\title{
Development of effective potentials for complex plasmas
}

\author{
T.S. Ramazanov , K.N. Dzhumagulova iD, M.T. Gabdullin iD , \\ Zh.A. Moldabekov iD and T.N. Ismagambetova* \\ Institute of Experimental and Theoretical Physics, Al-Farabi Kazakh National University, \\ 71, al-Farabi Ave, 050040, Almaty, Kazakhstan \\ *e-mail: ismagambetova@physics.kz
}

\begin{abstract}
In this article the review of interaction potentials for compound particles (i.e. atoms, clusters etc.) of complex plasmas that have been derived and developed by academician Fazylkhan Baimbetov and his students over the past 25 years is given. Complex plasmas such us dense non-ideal plasmas, partially ionized plasmas, dusty plasmas are considered. We consider effective interaction potentials for classical non-ideal plasmas, semiclassical weakly coupled plasmas, and quantum plasmas. The effective potentials include relevant effects of non-idelaity and quantum degeneracy. These effective potentials are for equilibrium and stationary out-of equilibrium state of plasmas. In the latter case, the effect of dynamical screening resulting in oscillatory pattern of the potential due to formation of the wakefield considered in both classical and quantum plasmas is taken into account. There appears anomalous behavior of the wakefield in quantum plasmas. Atoms and dust particles are considered to be compound particles with allowance for polarization, i.e acquiring a dipole moment. The screening of the field of such dipoles are considered making use the multipole expansion of the screened Coulomb potential. Additionally, the discussion of these results comparing to works of researchers from elsewhere is presented. The further development of the effective potential theory should determine what effect the plasma streaming will have on transport properties such as viscosity and diffusion of non-ideal plasmas.
\end{abstract}

Key words: effective potentials, pseudopotential model, polarization effect, complex plasma.

PACS numbers: 52.27.Lw, 52.65.- $-\mathrm{y}$

\section{Introduction}

Over the last several decades, researchers from various countries have shown increased interest in non-ideal plasmas; where the latter is characterized by importance of interparticle interactions. Initially, interest in non-ideal plasmas was due to the nuclear defense projects in several countries. Later, the research in non-ideal plasmas has been continuously fueled by the national ignition campaign in the US as well as other technological applications, such as pulsed MHD generators, rocket engines with a gas-phase reactor and plasmatrons. Nowadays, the interest in non-ideal plasmas is boosted by the laser-plasma experiments allowing to study the extreme state of matter in laboratory. This paves the way to investigate the properties of state of matter in various astrophysical objects (white dwarfs, giant planets etc.) in unprecedented details.

Besides that, large variety of scientific and technological problems is associated with strongly coupled dusty plasmas. For an adequate study of the properties of plasmas of such complex composition as dusty plasmas and non-ideal plasmas in general, it is of utmost importance to know the interaction potentials of the components of the system. The important physical effects that we should be taken into account are collective effects (higher-order correlations, screening, the interaction of a large number of particles, etc.) and quantum-mechanical effects (diffraction, symmetry, etc.).

\section{Pseudopotential models of the interaction of particles of classical non-ideal plasmas}

In plasmas, it is necessary to take into account the effects associated with the simultaneous interaction of a large number of charged particles due to long-range nature of the Coulomb interaction. As was expressed in F. Baimbetov's doctoral dissertation [1], higher order correlations can be taken into account not only in the particle 
distribution functions, but also in the potential of their interaction. As it is known, taking into account pair correlations in a rarefied system leads to the well-known Debye-Hückel potential. With increasing density, the average distance between particles decreases. In this case, the particles spend more and more time interacting with each other (the Klimontovich "delay" effect), the average particle interaction energy increases and the plasma properties can no longer be described in terms of pair correlations. Under these conditions, higher order correlations at large and quantum effects at small distances can be taken into account in the potential of interparticle interactions.

\subsection{The equation for the effective potential (pseudopotential) of dense plasmas [2,3]}

As already noted, in highly ionized plasmas at large distances between particles the interparticle interaction potential is different from the Coulomb interaction due to the effect of screening. In this regard, a method of computing a potential that takes into account collective (correlation) effects at large distances it is of great interest. The Bogolyubov chain for the equilibrium distribution functions [4] was taken as such a method and an equation for the effective potential (pseudopotential) was obtained on its basis $\tilde{\Phi}_{q k}$, taking into account the simultaneous correlation of particles in the framework of pair additivity [3]:

$$
\begin{gathered}
\Delta_{i} \sum_{j=1}^{S}{ }^{\prime} \tilde{\Phi}_{q k}=-4 \pi \sum_{j=1}^{S}{ }^{\prime} \delta\left({ }^{k} \vec{r}_{j}-{ }^{q} \vec{r}_{i}\right) e_{k} e_{q}+ \\
+\sum_{j=s+1}^{N} A_{k} \int\left(\Delta_{i} \Phi_{i j}\right) \exp \left\{-\frac{\tilde{\Phi}_{q k}\left({ }^{q} \vec{r}_{i,}{ }^{k} \vec{r}_{j}\right)}{\Theta}\right\} \\
\nabla_{j} \exp \left[-\frac{\sum_{l=1}^{S} / \tilde{\Phi}_{q k}}{\Theta}\right] d^{k} \vec{r}_{j}
\end{gathered}
$$

here $\Phi_{i j}$ is the microscopic Coulomb potential, $\Theta=k_{B} T$ and $q, k$ are the types of particles. In the approximation of pair correlations ( $S=2$ ), equation (1) leads to the Debye-Hückel potential as expected. In the three-particle correlations approximation, after symmetrizing and integrating the individual terms in (1), we have [3]:

$$
\Delta \Psi=\Psi \pm \Psi^{2}
$$

where $\Psi(R)$ is the effective potential in units of; $\Theta=k_{B} T, R=r / r_{D} ; r_{D}-$ Debye radius; $\Delta-$ Laplace operator. The minus sign corresponds to the interaction of the likewise charges, and the plus sign corresponds to the interaction of oppositely charged plasma particles.

\subsection{Classical dense plasma model}

To build a model of classical dense plasmas, it is necessary to take into account that

at small distances, the interaction between particles is described by the Coulomb potential, and at large distances it is equal to zero:

$$
\begin{gathered}
\frac{d^{2} \phi}{d r^{2}}+\frac{2}{r} \frac{d \phi}{d r}-\frac{\phi}{r_{D}^{2}}= \pm \frac{\phi^{2}}{r_{D}^{2} k_{B} T}, \\
\phi(r \rightarrow 0)=\phi_{\text {coul }}, \quad \phi(r \rightarrow \infty)=0
\end{gathered}
$$

here $\varphi=\Psi \cdot \Theta$

The boundary value problem (3) is nonlinear; therefore, it is solved numerically. However, in a fairly wide range of the parameter, the numerical solution (3) is approximated with a high degree of accuracy by the following interpolation formula $[3,5]$ :

$$
\Psi(R)=\frac{\gamma}{R} e^{-R} \frac{1+\frac{\gamma}{2} f(R)}{1+c(\gamma)} .
$$

where $f(R)=\left(e^{-\sqrt{\gamma} R-1}-1\right)\left(1-e^{-2 R}\right) / 5$ and $c(\gamma)$-a coefficient that depends on $\gamma$.

\subsection{Weakly coupled plasma model}

At parameters of weakly coupled classical plasmas, i.e. when, $\gamma=(Z e)^{2} / r_{D} k_{B} T<1$ for problem (3), we can construct an asymptotic expansion [6]:

$$
\begin{aligned}
& \Psi(R)=\gamma\left[1+\frac{\gamma}{2}\left\{\left(\operatorname{Ei}\left(-R_{0}\right)-\operatorname{Ei}(-R)\right)-\right.\right. \\
& \left.\left.-\left(\operatorname{Ei}\left(-3 R_{0}\right) e^{2 R_{0}}-\operatorname{Ei}(-3 R) e^{2 R}\right)\right\}\right] \frac{e^{-R}}{R}
\end{aligned}
$$


Here $E i(x)$ - integral exponential function, $R_{0}-$ the distance of closest approach of the particles.

\section{Effective potentials for semiclassical plasmas}

The inclusion of quantum effects in the interaction of charged particles allows us to solve the problem of divergence at small distances. By defining the quantum mechanical Sleter sum of particles interacting via Coulomb potential and comparing it with the classical Boltzmann factor, an analytical expression for the potential was obtained in $[7,8]$ :

$$
\begin{gathered}
\varphi_{a b}=\frac{e^{2}}{r}\left(1-\exp \left(-\frac{r}{\lambda_{a b}}\right)\right)+ \\
+\delta_{a e} \delta_{b e} k_{B} T \ln 2 \exp \left(-\frac{r^{2}}{\lambda^{2}{ }_{e e} \pi \ln 2}\right)
\end{gathered}
$$

where $\lambda_{a b}=\hbar /\left(2 \pi \mu_{a b} k_{B} T\right)^{1 / 2} \delta_{a e}-$ de Broglie wavelength, $\mu_{a b}$ - reduced mass of interacting particles, $\hbar$ - Planck's constant.

Potential (6) is widely known as the Deutsch potential and is used to study the physical properties of a semiclassical plasma. It should be noted that this potential takes into account only quantum effects of diffraction and symmetry, but does not take into account collective effects (for example, screening). Therefore, there is a need to obtain an effective interaction potential of particles of a dense quasiclassical plasma that takes into account both of the above effects.

In Ref. [9], pseudopotentials of the interaction of charged particles were obtained, which take into account the quantum-mechanical effects of diffraction at short distances and the effects of screening of the field at large distances. For this purpose, the dielectric response functions were used. To take into account quantum effects, the Deutsch potential with the diffraction part was used as the interaction micropotential [7]:

$$
\varphi_{a b}=\frac{e^{2}}{r}\left(1-\exp \left(-\frac{r}{\lambda_{a b}}\right)\right)
$$

and Coulomb potential was used as the micropotential of ion interaction.
The analytical expression for the electronelectron and electron-ion potential has the following form:

$$
\Phi_{\alpha \beta}=\frac{Z_{\alpha} Z_{\beta} e^{2}}{\sqrt{1-4 \lambda_{\alpha \beta}^{2} / r_{D}^{2}}}\left(\frac{e^{-B r}}{r}-\frac{e^{-A r}}{r}\right) .
$$

where $A^{2}=\frac{1}{2 \lambda_{\alpha \beta}^{2}}\left(1+\sqrt{1-\lambda_{\alpha \beta}^{2} / r_{D}^{2}}\right)$ and.

$$
B^{2}=\frac{1}{2 \lambda_{\alpha \beta}^{2}}\left(1-\sqrt{1-\lambda_{\alpha \beta}^{2} / r_{D}^{2}}\right)
$$

An expression was also obtained for the effective potential of ion-ion interaction $[10,11]$ :

$$
\Phi_{i i}(r)=\frac{Z_{i} Z_{i} e^{2}}{\sqrt{1-4 \lambda_{e i}^{2} / r_{D}^{2}}}\left(C_{1} \frac{e^{-B r}}{r}-C_{2} \frac{e^{-A r}}{r}\right),
$$

where

$$
\begin{aligned}
& C_{1}=\frac{1}{2}\left(1+\sqrt{1-\lambda_{e i}^{2} / r_{D}^{2}}\right), \\
& C_{2}=\frac{1}{2}\left(1-\sqrt{1-\lambda_{e i}^{2} / r_{D}^{2}}\right)
\end{aligned}
$$

The obtained effective potentials (8) and (9) are valid in the region of weakly degenerate plasma. These potentials were used in analytical calculations and computer simulations of the properties of a strongly coupled semiclassical plasma.

\section{Effective interparticle interaction potentials of partially-ionized dense plasmas taking into account polarization effects}

As the micropotential of interaction for electron - electron in the first case, as well as for electrondipole, dipole-dipole pairs, in the second case, we take the Deutsch micropotential (7). Consider a system of electrons and dipoles. A set of microscopic potentials is as follows:

$$
\begin{gathered}
\varphi_{e e}=\frac{e^{2}}{r}\left(1-\exp \left(-\frac{r}{\lambda}\right)\right) \\
\varphi_{e s}=-\frac{e Q_{s}(\nabla)}{r}, \\
\varphi_{s s}=-\frac{\left(Q_{s}(\nabla)\right)^{2}}{r},
\end{gathered}
$$


where- $\lambda=\hbar / \sqrt{\left(2 \pi m_{e} k_{A} T\right)}$ thermal de Broglie wavelength of electrons.

Using the indicated micropotentials and performing the inverse Fourier transform, we obtain the screened potential for the interaction of the electron - atom pair when [12]:

$$
\begin{gathered}
\Psi_{e s}(r)=-\frac{e(\vec{P} \vec{\nabla})}{r \sqrt{1-4 k^{2} / m^{2}}} \times \\
\times\left(e^{-B r}\left(1-\frac{B^{2}}{m^{2}}\right)-e^{-A r}\left(1-\frac{A^{2}}{m^{2}}\right)\right)
\end{gathered}
$$

where

$$
\begin{aligned}
& A^{2}=\frac{1}{2 \hbar^{2}}\left(1+\sqrt{1-4 \hbar^{2} / r_{D}^{2}}\right) \\
& B^{2}=\frac{1}{2 \hbar^{2}}\left(1-\sqrt{1-4 \hbar^{2} / r_{D}^{2}}\right)
\end{aligned}
$$

are coefficients depending plasma density and temperature.

In the limiting case $\lambda \ll r_{D}$ potential (14) transforms into the well-known interaction potential of an isolated atom and an electron:

$$
\Phi_{e s}=-\frac{e^{2} \alpha}{2 r^{4}}
$$

Finally, we consider a system of electrons and dipoles with the following micropotentials for electron-electron, electron-dipole, dipole-dipole pairs:

$$
\Phi_{e e}=\frac{e^{2}}{r}\left(1-\exp \left(-\frac{r}{\lambda}\right)\right)
$$

$$
\begin{gathered}
\Phi_{e s}=-\frac{e Q_{s}(\nabla)}{r}\left(1-e^{-r / \hbar}\right) ; \\
\Phi_{s s}=\frac{\left(Q_{s}(\nabla)\right)^{2}}{r}
\end{gathered}
$$

where it is assumed that in the interaction of an atom with an electron, diffraction effects are also taken into account. For this case, the following expression of the screened potential was obtained [12]:

$$
\begin{aligned}
& \Psi_{e s}(r)=-\frac{e^{2} \alpha}{2 r^{4} \sqrt{1-4 \hbar^{2} / r_{D}{ }^{2}}} \times \\
& \times\left(e^{-B r}(1+B r)-e^{-A r}(1+A r)\right)^{2}
\end{aligned}
$$

In the limit $\lambda \ll r_{D}$, potential (19) goes over to the following formula:

$$
\Phi_{e s}(r)=-\frac{e^{2} \alpha}{2 r^{4}}\left(1-e^{r / \hbar}\left(1+\frac{r}{\lambda}\right)\right)^{2}
$$

This suggests that at small distances, potential (20) tends to a finite value and agrees well with the semi-empirical Buckingham potential [13].

We note that in $[14,15]$ an alternative approach was developed for obtaining effective potentials based on the linearized Poisson - Boltzmann equation, which follows from (1). One of the simplest options for taking into account interparticle interactions is a self-consistent chemical model, first proposed in [14]. It is entirely based on the renormalization procedure of particle interactions [15], which leads to the following generalized Poisson-Boltzmann equation for the macroscopic potential of interaction of particle species and, taking into account collective events in the medium

$$
\Delta_{i} \Phi_{a b}\left(\mathbf{r}_{i}^{a}, \mathbf{r}_{j}^{b}\right)=\Delta_{i} \varphi_{a b}\left(\mathbf{r}_{i}^{a}, \mathbf{r}_{j}^{b}\right)-\sum_{c} \frac{n_{c}}{k_{B} T} \int \Delta_{i} \varphi_{a c}\left(\mathbf{r}_{i}^{a}, \mathbf{r}_{k}^{b}\right) \Phi_{c b}\left(\mathbf{r}_{j}^{b}, \mathbf{r}_{k}^{c}\right) d \mathbf{r}_{k}^{c}
$$

Here $\varphi_{a b}\left(\mathbf{r}_{i}^{a}, \mathbf{r}_{j}^{b}\right)$ denotes the genuine microscopic interaction potential, $\mathbf{r}_{i}^{a}$ stands for the radius vector of the $i$ 'th particle with $\Delta_{i}$ being the corresponding Laplace operator and $n_{c}$ signifies the number density of particles of sort $c$. Note that above and everywhere below the summation is implied over the repeated subscripts of particle species.

\section{Semiclassical effective interaction potentials of ions in quantum plasmas [16, 17]}

At high enough plasma densities, it becomes necessary to describe the system on the basis of the effective interaction potential of the ions, taking into account their wave nature (quantum diffraction effect) at small distances. Taking into account the wave nature of the ions should lead to a finite value of the effective potential at zero and would allow for 
the implementation of a semiclassical description of the ionic subsystem. The effective interaction potential, taking into account the wave nature of the ion at small interparticle distances, can be obtained using the dielectric response function method:

$$
\Phi_{i i}(\vec{r})=\int \frac{\mathrm{d}^{3} k}{2 \pi^{2}} \frac{Q_{i}^{2}}{k^{2} \varepsilon(\vec{k}, \omega=0)} \exp (i \vec{k} \vec{r}),
$$

here $Q_{i}$ is the charge of the ion. For this, in equation (21), instead of the Coulomb potential, it is necessary to use a semiclassical micropotential, which has a finite value for $r=0$, for example, the Deutsch potential (7) with a De Broglie wavelength for ions. By calculating the dielectric response function in the random phase approximation in the long-wavelength limit, we obtain the following effective semiclassical interaction potential of ions in a dense two-component plasma with degenerate electrons:

$$
\begin{gathered}
\Phi_{i i}(r)=\frac{Q_{i}^{2}}{r} \times\left[\xi \exp \left(-\frac{r}{\lambda_{i i}}\right)+\right. \\
+\gamma \exp (-C r)+\alpha \exp (-A r)+\beta \exp (-B r)]
\end{gathered}
$$

where constants $\xi, \alpha, \beta, \gamma, A, B, C$ depends on plasma parameters.

It should be noted that in the limit $\lambda_{i i} \rightarrow 0$, the effective potential (22) transforms into the Stanton and Murillo potential [18]. If we additionally neglect the gradient correction $\widetilde{a}_{2}=0$, we obtain a screened potential of the Yukawa type. The analysis showed that the effective semiclassical potential (22), which takes into account the quantum diffracttion effect, has a finite value for, on the contrary, a Yukawa type potential tends to infinity as.

\section{Effective interaction potentials of particles of semiclassical two-temperature plasmas}

In many experiments where a dense plasma is obtained, the temperature of electrons and ions has various values for quite a long time. Thus, it is important to take into account the fact that the plasma is non-isothermal. Dense plasma means a plasma where the average interparticle distance is comparable to the thermal wavelength of de Broglie particles and there is a high probability of particle collisions with a close encounter, in which it becomes important to take into account the wave nature of the colliding particles due to quantummechanical effects such as diffraction and symmetry. These effects at small distances can be taken into account in the pair potential of particle interaction or micropotential. The electron-ion temperature can be expressed through the temperature of electrons and ions in the form, $T_{e i}=\sqrt{T_{e} T_{i}}$ so that $T_{\alpha \beta}=\sqrt{T_{\alpha} T_{\beta}}$ [19].

Using the method of the dielectric response function and (7) as the micropotential, we obtain the expression for the effective interaction potential of particles of a nonisothermal plasma:

$$
\begin{gathered}
\Phi_{\alpha \beta}(r)=\frac{Z_{\alpha} Z_{\beta} e^{2}}{r} \frac{1}{\gamma^{2} \sqrt{1-\left(2 k_{D} / \lambda_{e e} \gamma^{2}\right)^{2}}}\left(\left(\frac{1 / \lambda_{e e}^{2}-B^{2}}{1-B^{2} \lambda_{\alpha \beta}{ }^{2}}\right) \exp (-B r)-\right. \\
-\left(\frac{1 / \lambda_{e e}{ }^{2}-A^{2}}{1-A^{2} \lambda_{\alpha \beta}{ }^{2}}\right) \exp (-A r)-\frac{Z_{\alpha} Z_{\beta} e^{2}}{r} \frac{\left(1-\delta_{\alpha \beta}\right)}{1+C_{\alpha \beta}} \exp \left(-r / \lambda_{\alpha \beta}\right)
\end{gathered}
$$

here $. C_{\alpha \beta}=\frac{k_{D}^{2} \lambda_{\alpha \beta}^{2}-k_{i}^{2} \lambda_{e e}^{2}}{\lambda_{e e}^{2} / \lambda_{\alpha \beta}^{2}-1}$

The effective potential (23) describes the interaction for all pairs of particles of a nonisothermal plasma. From formula (23) we obtain an analytical expression for the effective potential of electron-electron interaction:

$$
\begin{aligned}
\Phi_{e e}(r)= & \frac{e^{2}}{\left(1+\lambda^{2}{ }_{e e} k_{i}^{2}\right) \sqrt{1-\left(2 k_{D} / \lambda_{e e} \gamma^{2}\right)^{2}}} \times \\
& \times \frac{(\exp (-B r)-r x p(A r))}{r}
\end{aligned}
$$

for the effective potential of ion-ion interaction: 


$$
\begin{gathered}
\Phi_{i i}(r)=\frac{Z_{i} Z_{i} e^{2}}{\gamma^{2} \sqrt{1-\left(2 k_{D} / \lambda_{e e} \gamma^{2}\right)^{2}} r} \times \\
\times\left(\exp (-B r)\left(\frac{1}{\lambda_{e e}{ }^{2}}-B^{2}\right)-\right. \\
\left.-\exp (-A r)\left(\frac{1}{\lambda_{e e}{ }^{2}}-A^{2}\right)\right)
\end{gathered}
$$

Finally, for the effective potential of ion-ion interaction we have:

$$
\begin{gathered}
\Phi_{e i}(r)=\frac{Z_{i} e^{2}}{\lambda_{e i}{ }^{2} \gamma^{2} \sqrt{1-\left(2 k_{D} / \lambda_{e e} \gamma^{2}\right)^{2}} r} \times \\
\times\left(\left(\frac{1 / \lambda_{e e}{ }^{2}-B^{2}}{1 / \lambda_{e i}{ }^{2}-B^{2}}\right) \times \exp (-B r)-\left(\frac{1 / \lambda_{e e}{ }^{2}-A^{2}}{1 / \lambda_{e i}{ }^{2}-A^{2}}\right) \times\right. \\
\times \exp (-A r)+\frac{e^{2}}{r} \frac{1}{1+C_{e i}} \exp \left(-r / \lambda_{e i}\right)
\end{gathered}
$$

The obtained potentials satisfy all limit transitions. In the absence of shielding , $k_{D} \rightarrow 0, k_{i} \rightarrow 0, k_{e} \rightarrow 0$ formulas (24), (25) and (26) are equal reads:

$$
\begin{gathered}
\Phi_{e e}(r)=\frac{e^{2}}{r}\left(1-\exp \left(-\frac{r}{\lambda_{e e}}\right)\right), \\
\Phi_{i i}(r)=\frac{Z_{i} Z_{i} e^{2}}{r}, \\
\Phi_{e e}=-\frac{Z_{i} e^{2}}{r}\left(1-\exp \left(-\frac{r}{\lambda_{e i}}\right)\right),
\end{gathered}
$$

respectively. These potentials coincides with micropotentials, which are used as initial ones. In the case $\lambda_{e e} \rightarrow 0, \lambda_{e i} \rightarrow 0$, the expression for the effective potential, formula (23) transforms into the formula for the screened Debye potential neglecting quantum effects. Under the condition $k_{i} \rightarrow 0$, the | effective potentials (24) - (26) are consistent with the potentials obtained in $[9,11]$. Note that under the condition $4 \lambda_{e e}^{2} k_{e}^{2}=1$ and $k_{i}=0$, the expression for the ion-ion potential takes the following simplified form:

$$
\Phi_{i i}(r)=\frac{Z_{i}^{2} e^{2}}{r}\left(1+\frac{r}{2 \sqrt{2} \lambda_{e e}}\right) \exp \left(-\frac{r}{\sqrt{2} \lambda_{e e}}\right)
$$

\section{Effective interaction potentials of compound particles in classical plasmas [20-22]}

In many practical problems, it is necessary to consider the interaction of charged particles taking into account their internal structure. Types of particles the internal structure of which is important to take into account for the correct description of the plasma, will be referred to as compound particles. For example, such particles include atoms and micro- or nano-sized dust particles of a complex plasma, which were also considered in [23-25]. Now we consider the potential energy of interaction of the one system of charges with another system of charges located at a distance $R$ from the first (a schematic explanation is shown in Fig. 1), introducing the dipole moment $\vec{d}_{2}=\sum e_{\alpha}{ }^{(2)} \vec{r}_{\alpha}{ }^{(2)}$ and the total charge $Q_{2}=\sum e_{\alpha}{ }^{(2)}$ of the second system of charges, we find:

$$
\Phi=\varphi_{1} Q_{2}+\operatorname{grad} \varphi_{1} \cdot \vec{d}_{2}
$$

Using Eq. (28), for system of particles interacting via Coulomb potential, we find known result:

$$
\begin{gathered}
\Phi=\frac{Q_{1} Q_{2}}{R}+\frac{\left(Q_{2} \vec{d}_{1}-Q_{1} \vec{d}_{2}\right) \vec{R}}{R^{3}}+ \\
+\frac{\left(\vec{d}_{1} \vec{d}_{2}\right) R^{2}-3\left(\vec{d}_{1} \vec{R}\right)\left(\vec{d}_{2} \vec{R}\right)}{R^{5}}
\end{gathered}
$$




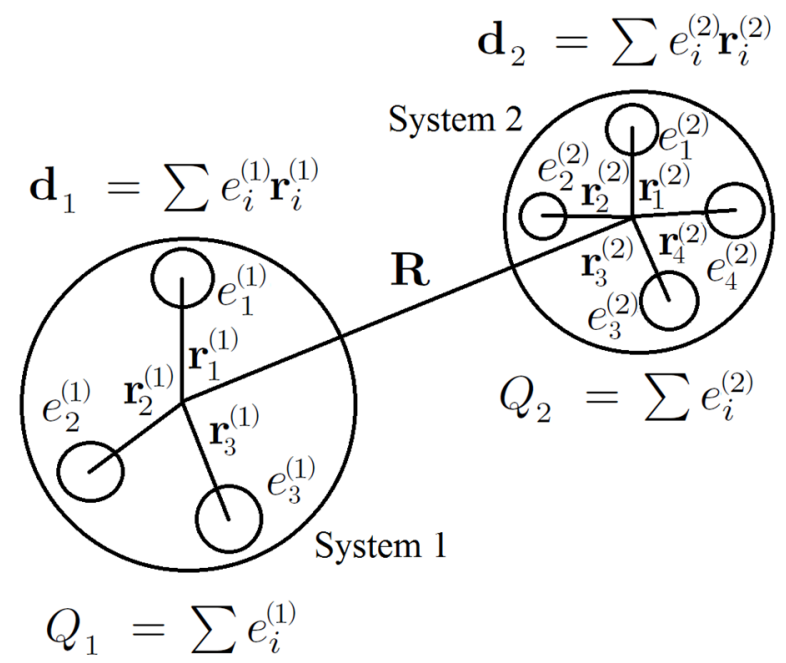

Figure 1 - Schematic explanation of the calculation of the interaction energy of two composite systems (particles)

Note that from expression (29) we can obtain all the basic formulas for potentials that are valid for various asymptotic cases [21]. We now consider the field of a composite particle in a polarizable medium.
In such a medium, the field of a single charged particle is determined by a formula of the type (21). Using the methodology described above, we obtain the following expression for the effective potential:

$$
\begin{aligned}
& \Phi=\frac{Q_{1} Q_{2}}{R} \exp \left(-R k_{s}\right)+\frac{\left(Q_{2} \vec{d}_{1}-Q_{1} \vec{d}_{2}\right) \vec{R}}{R^{3}}\left(1+R k_{s}\right) \exp \left(-R k_{s}\right)+ \\
& +\frac{\left(\vec{d}_{1} \vec{d}_{2}\right) R^{2}-\left(\vec{d}_{1} \vec{R}\right)\left(\vec{d}_{2} \vec{R}\right)\left(3+R k_{s}\right)+\left(\overrightarrow{d_{1}} \vec{R}\right)\left(\vec{d}_{2} \vec{R}\right) R k_{s}}{R^{5}} \exp \left(-R k_{s}\right)
\end{aligned}
$$

We consider some limiting cases of potential (30). In particular, the case when two composite particles have parallel dipole moments is of interest. For such particles from Eq. (30) we find:

$$
\Phi=\frac{Q^{2}}{R} \exp \left(-R k_{s}\right)+\frac{d^{2}}{R^{3}}\left(1+R k_{s}\right) \exp \left(-R k_{s}\right)
$$

where we set $d_{1}=d_{2}$.

In the case when the dust particles are located along the direction of the ion flux, from equation (30) we deduce:

$$
\begin{gathered}
\Phi=\frac{Q^{2}}{R} \exp \left(-R k_{s}\right)- \\
-\frac{2 d^{2}}{R^{3}}\left(1+R k_{s}+\frac{R^{2} k_{s}^{2}}{2}\right) \exp \left(-R k_{s}\right)
\end{gathered}
$$

As can be seen from Eq. (32), at long distances, attraction between like charged particles occurs along the direction of the ion flow. This behavior of dust particles was observed in experiments on dusty plasmas in a gas discharge of direct current. Note that the oscillatory nature of the effective dipole dipole interaction, responsible for the formation of ordered structures in a dusty plasmas, is also shown in Ref. [20].

For the case of the interaction of a charge with a dipole, we obtain:

$$
\Phi_{d-c h}=\frac{Q_{1} \vec{d}_{2} \vec{R}}{R^{3}}\left(1+R k_{s}\right) \exp \left(-R k_{s}\right)
$$

The interaction of two dipoles, , $Q_{1}=Q_{1}=0, \vec{d}_{1} \neq 0, \vec{d}_{2} \neq 0$ is described by the following formula: 


$$
\Phi_{d-d}=\frac{\left[\left(\vec{d}_{1} \vec{d}_{2}\right) R^{2}-\left(\vec{d}_{1} \vec{R}\right)\left(\vec{d}_{2} \vec{R}\right)\left(3+R k_{s}\right)\right]\left(1+R k_{s}\right)+\left(\vec{d}_{1} \vec{R}\right)\left(\vec{d}_{2} \vec{R}\right) R k_{s}}{R^{5}} \exp \left(-R k_{s}\right)
$$

\section{Effective interaction potentials of compound particles in quantum plasmas}

As it is known, in dense plasma, when the thermal wavelength of electrons $\lambda=\hbar /\left(2 \pi m_{e} k_{B} T\right)$ becomes comparable with the average interparticle distance, it is necessary to take into account the wave nature of electrons. Calculating the static dielectric function in the random phase approximation, we find the interaction energy of two composite particles in a dense plasma:

$$
\begin{gathered}
\Phi=\frac{Q_{1} Q_{2}}{R} f_{1}(R)+\frac{\left(Q_{2} \vec{d}_{1}-Q_{1} \vec{d}_{2}\right) \vec{R}}{R^{3}} f_{2}(R)+ \\
+\frac{\left(\vec{d}_{1} \vec{d}_{2}\right) R^{2} f_{2}(R)+\left(\vec{d}_{1} \vec{R}\right)\left(\vec{d}_{2} \vec{R}\right) f_{3}(R)}{R^{5}}
\end{gathered}
$$

where for convenience the following functions are introduced:

$$
\begin{gathered}
f_{1}(R)=\frac{\left(\exp (-B R)\left(\frac{1}{\lambda_{e e}^{2}}-B^{2}\right)-\exp (-A R)\left(\frac{1}{\lambda_{e e}^{2}}-A^{2}\right)\right.}{\gamma^{2} \sqrt{1-\left(2 k_{D} / \lambda_{e e} \gamma^{2}\right)^{2}}} \\
f_{2}(R)=\frac{\left(\exp (-B R)(1+R B)\left(\frac{1}{\lambda_{e e}^{2}}-B^{2}\right)-\exp (-A R)(1+R A)\left(\frac{1}{\lambda_{e e}^{2}}-A^{2}\right)\right.}{\gamma^{2} \sqrt{1-\left(2 k_{D} / \lambda_{e e} \gamma^{2}\right)^{2}}} \\
f_{3}(R)=\frac{1}{\gamma^{2} \sqrt{1-\left(2 k_{D} / \lambda_{e e} \gamma^{2}\right)^{2}}}\left(\begin{array}{l}
3 \exp (-B R)(1+R B)\left(\frac{1}{\lambda_{e e}^{2}}-B^{2}\right)+\exp (-B R) B^{2} R^{2}(1+R A)\left(\frac{1}{\lambda_{e e}^{2}}-B^{2}\right)- \\
-3 \exp (-A R)(1+R A)\left(\frac{1}{\gamma_{e e}^{2}}-A^{2}\right)-\exp (-A R) A^{2} R^{2}\left(\frac{1}{\lambda_{e e}^{2}}-A^{2}\right)
\end{array}\right)
\end{gathered}
$$

Effective potential (35) at $\lambda_{e e} \rightarrow 0$ and $\lambda \rightarrow 0$ reduces to the effective potentials (30).

9 Dynamically screened potential of an ion in a stationary nonequilibrium plasma $[26,27]$

It is known that often in experiments dense plasmas far from the equilibrium state are created. For instance, in experiments on the compression of matter by lasers and high-energy particle beams, a flow of particles of one type relative to particles of another type appears. In such a plasma, statically screened interaction potentials do not provide a satisfactory description. It is necessary to take into account the effect of dynamic screening. Let us consider the dynamically screened potential of an ion in a dense plasma with a density parameter $r_{s} \leq 1$, i.e., with a density $n>10^{24} \mathrm{~cm}^{-3}$. The electron degeneracy parameter is taken within $0.01 \leq \theta \leq 10$. The effects of non-ideality in the electronic subsystem are taken into account on the basis of the relationship between the collision frequency and the dielectric constant of Mermin: 


$$
\varepsilon_{M}(\vec{k}, \omega)=1+\frac{(\omega+i v)\left\lfloor\varepsilon_{R P A}(\vec{k}, \omega+i v)-1\right\rfloor}{\omega+i v\left\lfloor\varepsilon_{R P A}(\vec{k}, \omega+i v)-1\right\rfloor \times\left\lfloor\varepsilon_{R P A}(\vec{k}, 0)-1\right\rfloor^{-1}}
$$

Using relation (21) with dielectric function (39), dynamically screened ion-ion interaction potential in a stationary nonequilibrium plasma can be computed. A typical dynamically screened ion field is shown in Figure 2. For clarity, the "inverted" form of the potential is shown, that is, multiplied by -1 . The peak in Figure 2 (minimum of potential) behind an ion, relative to the flux of electrons, corresponds to the area where ions attracts to eachother.

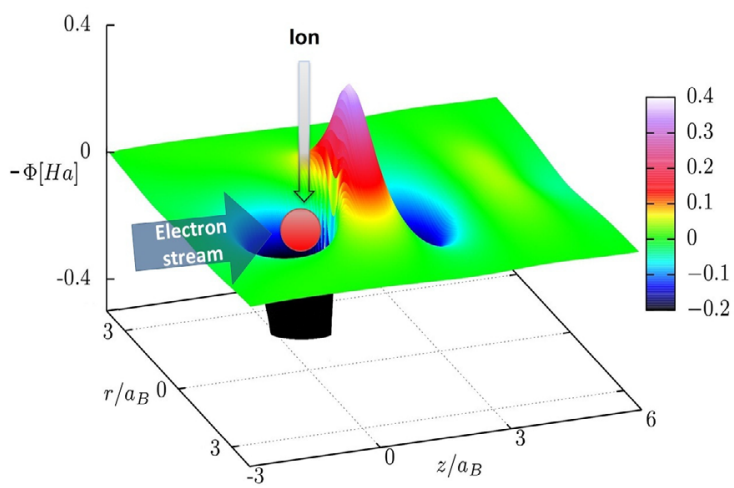

Figure 2 - Dynamically screened potential of an ion in a quantum plasma. The ion is located at the point $(r=0: z=0)$. The electron flux is directed from left to right. For purposes of illustration, the inverse of the ion potential is shown, i.e. multiplied by -1 .

\section{Conclusions and Outlook}

The overview of the results on the effective interaction potentials of plasma particles that have been obtained over the past 25 years by representatives of the academic school of plasma physics of academician Fazylkhan Baimbetov is given. These effective potentials have proven to provide adequate description of the various physical properties and processes occurring in a complex plasma.

Obtained effective potentials can be used for the computation of the various physical properties of plasmas. The transport properties can be found using the effective interaction potential for computation of the scattering cross section and relevant Coulomb logarithm. Furthermore, the scattering cross section can be used to compute the collision frequency and, thus, the temperature relaxation. Additionally, the knowledge of the collision frequency allows to investigate the optical properties of plasma using the relaxation time approximation for the closure of the one particle kinetic equation. Next, the effective interaction potentials can be used to compute structural properties, such as radial distribution function of particles and static structure factor, making use of the integral equations. Therefore, once radial distribution function is known, thermodynamic properties can be easily calculated.

Although non-ideal plasmas created in experiments are usually non-equilibrium, most of the works considered equilibrium non-ideal plasmas. The results on screening of the test charge in classical as well as quantum streaming plasmas clearly shows that dynamically screened potential can significantly deviate from that of in equilibrium plasmas. Therefore, it is interesting to study transport properties of particles in streaming plasmas. In other words, what is the effect of plasma streaming on such transport properties as viscosity and diffusion of non-ideal plasmas. It is anticipated that the further development of the effective potential theory should give an answer to this question.

\section{References}

1. Baimbetov, F. (1985) Transport processes and relaxation phenomena in plasma and dense gases. The dissertation for Doctoral degree in Physical and Mathematical Sciences. Tbilisi. [in russian].

2. Baimbetov, F. B., Ramazanov, T.S. (1990) Equilibrium properties of dense classical plasma. Computing Experiment Data. Thermophysics of high temperatures, 28, 3, 595-597 [in russian].

3. Baimbetov, F.B., Nurekenov, Kh.T., Ramazanov, T.S. (1995) Pseudopotential theory of classical non-ideal plasmas. Physics Letters A., 202, 211-214.

4. Bogolyubov, N. N. (1979) Problems of dynamic theory in statistical physics. M. Science. [in russian]. 
5. Baimbetov, F.B., Nurekenov, Kh.T., Ramazanov, T.S. (1996) Electrical conductivity and scattering sections of strongly coupled hydrogen plasmas. Physica A, 226, 181-190.

6. Baimbetov, F. B., Dzhumagulova, K.N., Ramazanov, T.S. (1995) On the thermodynamics of weakly non-ideal plasma. Thermophysics of high temperatures, 33, 644-646 [in russian].

7. Deutsch, C., Furutani ,Y., Gombert, M.M. (1981) Nodal expansions for strongly coupled classical plasmas. Phys. Rep., 69, 85-193.

8. Minoo, H., Gombert, M.M., Deutsch, C. (1981) Temperature-dependent Coulomb interactions in hydrogenic systems. Phys. Rev. A., 23, 924.

9. Ramazanov, T.S., Dzhumagulova, K.N. (2002) Effective screened potentials of strongly coupled semiclassical plasma. Phys. Plasmas., 9, 9, 3758.

10. Ramazanov, T.S., Dzhumagulova, K.N., Gabdullin, M.T. (2006) Microscopic and thermodynamic properties of dense semiclassical partially ionized hydrogen plasma. J. Phys. A: Math. Gen., 39, 4469.

11. Ramazanov, T.S., Dzhumagulova, K.N., Gabdullin, M.T. (2010) Effective potentials for ion-ion and chargeatom interactions of dense semiclassical plasma. Phys. Plasmas, 17, 042703.

12. Ramazanov, T.S., Dzhumagulova, K.N. and Omarbakieva, Y.A. (2005) Effective polarization interaction potentials “charge-atom" for partially ionized plasma. Phys. Plasmas 12, 9, 092702.

13. Redmer, R. (1999) Electrical conductivity of dense metal plasmas. Phys. Rev. E, 59, 1073.

14. Arkhipov, Y.V., Baimbetov, F.B., and Davletov, A.E. (2005) Ionization equilibrium and equation of state of partially ionized hydrogen plasmas: Pseudopotential approach in chemical picture. Phys. Plasmas, $12,082701$.

15. Baimbetov, F.B., Davletov, A.E., Kudyshev, Z.A., Mukhametkarimov, E.S. (2010) New Model of Dusty Plasma Particles Interaction. Contributions to Plasma Physics, 51, 6, 533-536.

16. Moldabekov, Zh., Schoof, T., Ludwig, P, Bonitz, M., and Ramazanov, T. (2015) Statically screened ion potential and Bohm potential in a quantum plasma. Phys. Plasmas, 22, 102104.

17. Moldabekov, Zh.A., Ramazanov, T.S., and Dzhumagulova, K.N. (2012) Pair Interaction Potential of Particles for Two-Component Plasma. Contrib. Plasma Phys., 52, 207-210.

18. Stanton, L.G. and Murillo, M.S. (2015) Unified description of linear screening in dense plasmas. Phys. Rev. E, 91, 033104.

19. Schoof, T., Groth, S., Vorberger, J., and Bonitz, M. (2015) Ab Initio Thermodynamic Results for the Degenerate Electron Gas at Finite Temperature, Phys. Rev. Lett., 115, 130402.

20. Ramazanov, T.S., Moldabekov, Zh.A., Dzhumagulova, K.N., and Muratov, M.M. (2011) Pseudopotentials of the particles interactions in complex plasmas. Phys. Plasmas, 18, 103705.

21. Ramazanov, T.S., Moldabekov, Zh.A., and Gabdullin, M.T. (2016) Multipole expansion in plasmas: Effective interaction potentials between compound particles. Physical Review E, 93, 053204.

22. Ramazanov, T. S., Kodanova, S.K., Daniyarov, T.T., and Moldabekov, Zh.A. (2011) Investigation an Effective Interaction Potential of Dust Particles in Nonideal Dusty Plasma. Contrib. Plasma Phys., 51, 6, 514-518.

23. Lapenta, G. (1995) Dipole moments on dust particles immersed in anistropic plasmas. Phys. Rev. Lett., 75, 4409-4412.

24. Shukla, P.K. and Eliasson, B. (2009) Colloquium: Fundamentals of dust-plasma interactions. Reviews of Modern Physics, 81, 25-44.

25. Sukhinin, G.I., and Fedoseev, A.V. (2010) Formation of a Trapped-Ion Cloud Around a Dust

26. Particle in Low-Density Plasma. IEEE Transactions on Plasma science, 38, 6, 2345-2352.

27. Moldabekov, Zh.A., Ludwig, P., Bonitz, M., and Ramazanov, T.S. (2015) Ion potential in warm dense matter: Wake effects due to streaming degenerate electrons. Phys. Rev. E, 91, 023102.

28. Moldabekov, Zh.A., Ludwig, P., Joost, J.P., Bonitz, M., Ramazanov, T.S. (2015) Dynamical Screening and Wake Effects in Classical, Quantum, and Ultrarelativistic Plasmas. Contrib. Plasma Phys., 55, 186. 